\title{
Low-cost electromyography: validity against a commercial system depends on exercise type and intensity
}

\author{
Paolo Tecchio, Andrea Monte, Paola Zamparo \\ Department of Neurosciences, Biomedicine and Movement Sciences, University of Verona, \\ Verona, Italy. \\ This article is distributed under the terms of the Creative Commons Attribution Noncommercial License (CC BY-NC 4.0) which permits \\ any noncommercial use, distribution, and reproduction in any medium, provided the original author(s) and source are credited.
}

\begin{abstract}
The aim of this study was to assess the validity of a custom-made low cost (LC) and a commercial surface EMG apparatus in controlled experimental conditions and different exercise types: maximal voluntary contractions (MVC) at 105, 90, 75, 60, 45 and $30^{\circ}$ knee angle and explosive fix-end contractions of the knee extensors $\left(75^{\circ}\right)$ at an isometric dynamometer. sEMG of vastus lateralis was recorded from the same electrodes simultaneously, then analyzed in the same way; sEMG were finally expressed in percentage of those collected at $75^{\circ} \mathrm{MVC}$. LC underestimated the sEMG signal at the more extended knee angles $\left(30-60^{\circ}\right)$, significant difference was observed only at $30^{\circ}$. In the explosive contractions no differences between devices were observed in average and peak sEMG, as well as in the time to peak and the activation time. Bland-Altman tests and correlation parameters indicate the LC device is not sensible enough to detect the time to peak and the peak values of the sEMG signal properly. Results suggest low-cost systems might be a valid alternative to commercial ones, but attention must be paid when analyzing rapid events.
\end{abstract}

Key Words: Open-source technology; EMG analysis; biomedical sensors; maximal voluntary contractions.

Eur J Transl Myol 31 (2): 9735, 2021 doi: 10.4081/ejtm.2021.9735

The electromyographic signal (EMG) is commonly defined as the electrical activity of a given muscle during contraction. EMG analysis is utilized to investigate nerve conduction, muscle firing rate, muscle fatigue or different fibers activation but also to detect how the muscle's electrical activity could be influenced by disease, injuries, or different scenarios, ${ }^{1,2}$ as well as modified by training., ${ }^{1,3}$ The EMG signal can be acquired in different ways, but the most commonly used method is surface electromyography (sEMG), which reflects the general electrical characteristics of the area where the electrodes are positioned. Due to its simplicity and portability, sEMG analysis can be performed by sport scientists and/or physical therapists in their daily work to study muscle activity among different populations or in the pre/post training periods. Nevertheless, sEMG analysis in sport teams or rehabilitation centers it is not widespread also because commercial devices are quite expensive, around $€ 15.000$ to $20.000 .{ }^{5}$ Nowadays, simple and inexpensive electronic microchips (such as the Arduino board) can be utilized to acquire biological signals, resulting in low-cost EMG apparatus. However, the validation of these prototypes is still limited. As an example, Supuk et al. ${ }^{6}$ recorded sEMG activity during walking from six lower limb muscles with a self-developed low-cost apparatus compared to sEMG data reported in the literature; visual comparison of measured and reference envelope curves indicated a fairly good overlap for all muscles. More recently, Heywood S et al. ${ }^{7}$ determined the concurrent validity of a low-cost sEMG system and a commercial device by positioning two sets of electrodes end to end along the vastus lateralis muscle and asking the volunteers to perform different exercises (isometric contractions, knee extension, squatting, stepping and jumping); the relative agreement between the systems was found to be fairly good but to depend on the exercise type, on the sEMG parameter investigated (peak or mean muscle activation and contraction duration), and the correlation statistic used. ${ }^{5}$ Fuentes Del Toro et al. ${ }^{5}$ recently validated a lowcost apparatus against a commercial one by positioning two sets of electrodes side by side on the rectus femoris muscle and asking the volunteers to perform different isometric and dynamic exercises (squat, lounge, knee extension and vertical jumps); also in this study the relative agreement between the systems was found to be fairly good but with differences among exercise types 
and concurrent validity was found to depend on the correlation statistic used. ${ }^{5}$ It could then be argued whether the concurrent validity between low cost and commercial systems reported in the literature could be attributed to: i) the exercise type (isometric vs. dynamic), ii) the statistical analysis performed and/or iii) differences in data collection/analysis. The aim of this study was to evaluate the concurrent validity of a custommade and a commercial sEMG device under strictly controlled conditions, by taking into account possible confounding factors. The exercises of choice were: i) maximal voluntary isometric contractions (knee extension MVC at different joint angles) and ii) explosive fix-end contractions at an isometric dynamometer. To take into account the first issue (does concurrent validity depend on exercise type?), we decided to investigate maximal voluntary contractions (5 s duration) and explosive fix-end contractions. According to the forcelength relationship, differences in force (or torque) and in sEMG activity could be observed as a function of knee angle both during fast and during ramp contractions. Lanza et al. ${ }^{8,9}$ recently reported that the sEMG activity during maximal isometric contractions is the highest at the most flexed knee angles and decreases when joint extension increases. Thus, by changing the knee angle, it is possible to investigate the concurrent validity of the two systems at different EMG amplitudes but with small amplitude changes. Moreover, Heywood et al. ${ }^{7}$ observed that concurrent validity differs according to the temporal characteristics of the signal (whether of the sEMG signal we consider peak value, average value or its duration. Therefore, for the explosive fix-end contractions we decided to compare the EMG values (low-cost vs. commercial apparatus) also in terms of temporal parameters, investigating the duration of explosive contraction and time to peak of the EMG signal. To take into account the second issue (does concurrent validity depend the statistical analysis performed?), we utilized different statistical methods to assess concurrent validity between devices (RM ANOVA, Bland-Altman plots, Interclass Correlation Coefficient (ICC) and cross correlation analysis) and we investigated whether these methods give consistent results. In regard to the third issue (does concurrent validity depend on data acquisition and analysis?), in previous studies the EMG electrodes were positioned in a different, although close, portion of the investigated muscle. Indeed, major source of difference in signal amplitude is the position of the electrodes since the relative alignment and movement of the muscle fibers, the location of the innervation zone and cross talk between muscles influence EMG signal and quality. Therefore, we decided to derive the sEMG signal from the very same electrodes and we used the same data processing for the raw data acquisition from both EMG apparatuses. Due to the differences in signal gain between apparatuses and the expected differences in EMG amplitude among joint angles, it could be hypothesized that the differences between commercial and low-cost EMG devices could be exacerbated at specific joint angles where the EMG amplitude is the lowest. Finally, commercial and low-cost EMG apparatuses have different resolution of the AD converter (10 vs. 16 bits); it can thus be hypothesized that low-cost EMG apparatus could not detect rapid and short events, such as time to peak of the EMG signal.

\section{Materials and Methods}

\section{Participants}

Thirteen healthy active subjects (8 males, 5 females) without neuromotor impairments or injury in the right leg in the last year participated in this study (26.5 \pm 5.2 years of age; $68.7 \pm 16.5 \mathrm{~kg}$ body mass; $1.72 \pm 0.10 \mathrm{~m}$ stature). The study agreed with the Declaration of Helsinki for the study on human subjects. The local ethical committee approved the experimental protocol (2020-UNVRCLE161), and all subjects gave their written informed consent.

\section{Data collection}

Participants were fixed to a dynamometer (Cybex NORM) with a trunk and pelvic strap, and hold the arms crossed in front of their chest. The hip joint angle was set at $80^{\circ}\left(0^{\circ}\right.$ refers to supine position). The knee joint rotation was aligned with the dynamometer axis of rotation during a maximal voluntary contraction (MVC) at $60^{\circ}$ of knee flexion. ${ }^{10-13}$ Each participant performed a series of MVC with the right leg at 105, 90, 75, 60, 45 and $30^{\circ}\left(0^{\circ}=\right.$ knee fully extended $)$; two minutes of recovery time was interposed between contractions. The participants were instructed to contract as hard as possible from rest to maximum, but with a gradual increase (according to Maffiuletti et al. ${ }^{1}$; the duration of these contractions was $5 \mathrm{~s}$. At each angle the contractions were repeated twice, and their average value was used for further analysis. After the MVCs, each subject performed 5 maximal explosive contractions with 30 s of recovery in between (at $75^{\circ}$ knee angle); their average value was used for further analysis. During these contractions, the subjects were instructed to contract as hard and as fast as possible (according to Maffiuletti et al. ${ }^{1}$ ). Two surface electrodes (FIABC), Florence, Italy, Ag/AgCl, disc diameter ) were placed according to SENIAM guidelines on the right vastus lateralis muscle: ${ }^{14}$ the skin area was first shaved with a razor blade and then carefully cleaned up with alcohol. The (surface) sEMG signal was recorded from the same two surface electrodes with a wireless commercial device (ZW, ZeroWire, Cometa $₫$ srl, Italy) and a low-cost (LC) system at the same sample frequency $(1000 \mathrm{~Hz})$. Inter-electrode distance was $26 \mathrm{~mm}$. Two insulated crocodile splitter cables were used; the crocodile connectors were attached to the surface electrodes, while the extremities of the splitter were connected to both EMG devices. Finally, a third (ground) electrode was attached to the patellar tendon for the LC system only (see Figure S1 of the supplementary material). ${ }^{14}$ With this arrangement, both devices 
Table 1. sEMG activity (RMS and temporal parameters) during maximal voluntary and explosive fix-end contractions at different knee angles, as determined by means of a low cost (LC) and a commercial (ZW) EMG apparatus. Data are means $\pm S D$.

\begin{tabular}{|c|c|c|c|c|}
\hline \multicolumn{5}{|c|}{ Maximal voluntary contractions } \\
\hline & knee angle & LC & ZW & $\mathbf{P}$ \\
\hline & $105^{\circ}$ & $105.8 \pm 19.3$ & $107.3 \pm 22.8$ & 0.99 \\
\hline & $90^{\circ}$ & $111.7 \pm 21.0$ & $111.0 \pm 19.0$ & 0.99 \\
\hline RMS & $75^{\circ}$ & $100 \pm 0$ & $100 \pm 0$ & \\
\hline \multirow[t]{3}{*}{ (steady state) } & $60^{\circ}$ & $85.1 \pm 13.1$ & $90.5 \pm 17.6$ & 0.15 \\
\hline & $45^{\circ}$ & $73.4 \pm 19.7$ & $81.4 \pm 22,7$ & 0.054 \\
\hline & $30^{\circ}$ & $75.6 \pm 25.4$ & $87.7 \pm 32.7$ & 0.0008 \\
\hline \multicolumn{5}{|c|}{ Fix-end explosive contractions } \\
\hline & knee angle & LC & ZW & $\mathbf{P}$ \\
\hline Peak RMS & $75^{\circ}$ & $109.6 \pm 33.8$ & $101.1 \pm 28.7$ & 0.38 \\
\hline Mean RMS & $75^{\circ}$ & $90.8 \pm 24.6$ & $92.5 \pm 24.0$ & 0.68 \\
\hline TTP (ms) & $75^{\circ}$ & $137.7 \pm 36.7$ & $146.0 \pm 38.7$ & 0.38 \\
\hline AT (s) & $75^{\circ}$ & $1.5 \pm 0.3$ & $1.5 \pm 0.3$ & 0.14 \\
\hline
\end{tabular}

Footnote: TTP: time to peak; AT: activation time; P: $P$ values at paired t-test.

collected the sEMG signal from the same location at the same time and at the same sample frequency. The lowcost (LC) system was built with an Arduino Uno Ethernet Board (Arduino $\subset$, Italy) and an integrated signal conditioning block for biological signals (AD8232:

https://www.analog.com/media/en/technicaldocumentation/data-sheets/ad8232.pdf).

The LC system saved data automatically in a SD card, in order to avoid a possible bias due to the transmission from the apparatus to the PC through the serial/USB port. The signal gain and the sample rate of the two systems were: $60 \mathrm{~dB}$ and 16 bits for $\mathrm{ZW}$ and $40 \mathrm{~dB}$ (at $1000 \mathrm{~Hz}$ ) and 10 bits for LC; the operating voltage was: $4 \mathrm{~V}$ for $\mathrm{ZW}$ and $3.3 \mathrm{~V}$ for LC. The sampling rate affects the resolution of the device: the commercial system can resolve $65536\left(2^{16}\right)$ levels, whereas the LC systems has only 1024 of ideal range $\left(2^{10}\right)$. Thus, the commercial system can detect more accurately small differences in

Table 2. Correlational analysis: Bland Altman test (bias, SD and confidence intervals: CI), intraclass correlation coefficient (ICC) and goodness fit $\left(R^{2}\right)$ during maximal voluntary and explosive fix-end contractions at different knee angles, as determined by means of a low cost (LC) and a commercial (ZW) EMG apparatus. See Table 1 and text for details.

\begin{tabular}{|c|c|c|c|c|c|c|c|c|}
\hline \multicolumn{9}{|c|}{ Maximal voluntary contractions } \\
\hline & \multirow[b]{2}{*}{ KA } & \multirow{2}{*}{$\begin{array}{c}\text { Bias } \\
\text { ZW-LC }\end{array}$} & \multirow[t]{2}{*}{ SD } & \multicolumn{2}{|c|}{$\mathbf{C l}$} & \multicolumn{2}{|c|}{ ICC } & \multirow[t]{2}{*}{$\mathbf{R}^{2}$} \\
\hline & & & & from & to & mean & range & \\
\hline & $105^{\circ}$ & 1.57 & 12.8 & -23.5 & 26.7 & $0.82 * * *$ & $0.50-0.94$ & $0.68 * *$ \\
\hline & $90^{\circ}$ & 0.66 & 6.6 & -12.4 & 13.7 & $0.94 * * *$ & $0.83-0.98$ & $0.90 * *$ \\
\hline RMS & $75^{\circ}$ & & & & & & & \\
\hline \multirow{3}{*}{ (steady state) } & $60^{\circ}$ & -5.39 & 7.6 & -20.2 & 9.5 & $0.88 * * *$ & $0.65-0.96$ & $0.85^{* *}$ \\
\hline & $45^{\circ}$ & -8.04 & 7.5 & -22.7 & 6.6 & $0.94 * * *$ & $0.81-0.98$ & $0.90 * *$ \\
\hline & $30^{\circ}$ & -12.17 & 22.0 & -55.3 & 31 & $0.71 * *$ & $0.30-0.90$ & $0.55^{*}$ \\
\hline \multicolumn{9}{|c|}{ Fixed-end explosive contractions } \\
\hline & & Bias & SD & \multicolumn{2}{|c|}{$\mathrm{Cl}$} & \multicolumn{2}{|c|}{ ICC } & $\mathbf{R}^{2}$ \\
\hline & KA & ZW-LC & & from & to & mean & range & \\
\hline Peak RMS & $75^{\circ}$ & -8.52 & 33.7 & -74.6 & 57.6 & 0.42 & $-0.14-0.78$ & 0.18 \\
\hline Mean RMS & $75^{\circ}$ & 1.67 & 14.4 & -26.5 & 29.9 & $0.85^{* * *}$ & $0.52-0.94$ & $0.68 * *$ \\
\hline TTP (ms) & $75^{\circ}$ & 8.24 & 32.6 & -55.6 & 72.1 & $0.63 * *$ & $0.14-0.87$ & $0.39 \#$ \\
\hline AT (s) & $75^{\circ}$ & 0.05 & 0.1 & -0.16 & 0.26 & $0.95 * * *$ & $0.83-0.94$ & $0.90 * *$ \\
\hline
\end{tabular}

Footnote: KA: knee angle; TTP: time to peak; AT: activation time; \# $p<0.05 ;{ }^{*} p<0.01$; **: $p<0.001$ 

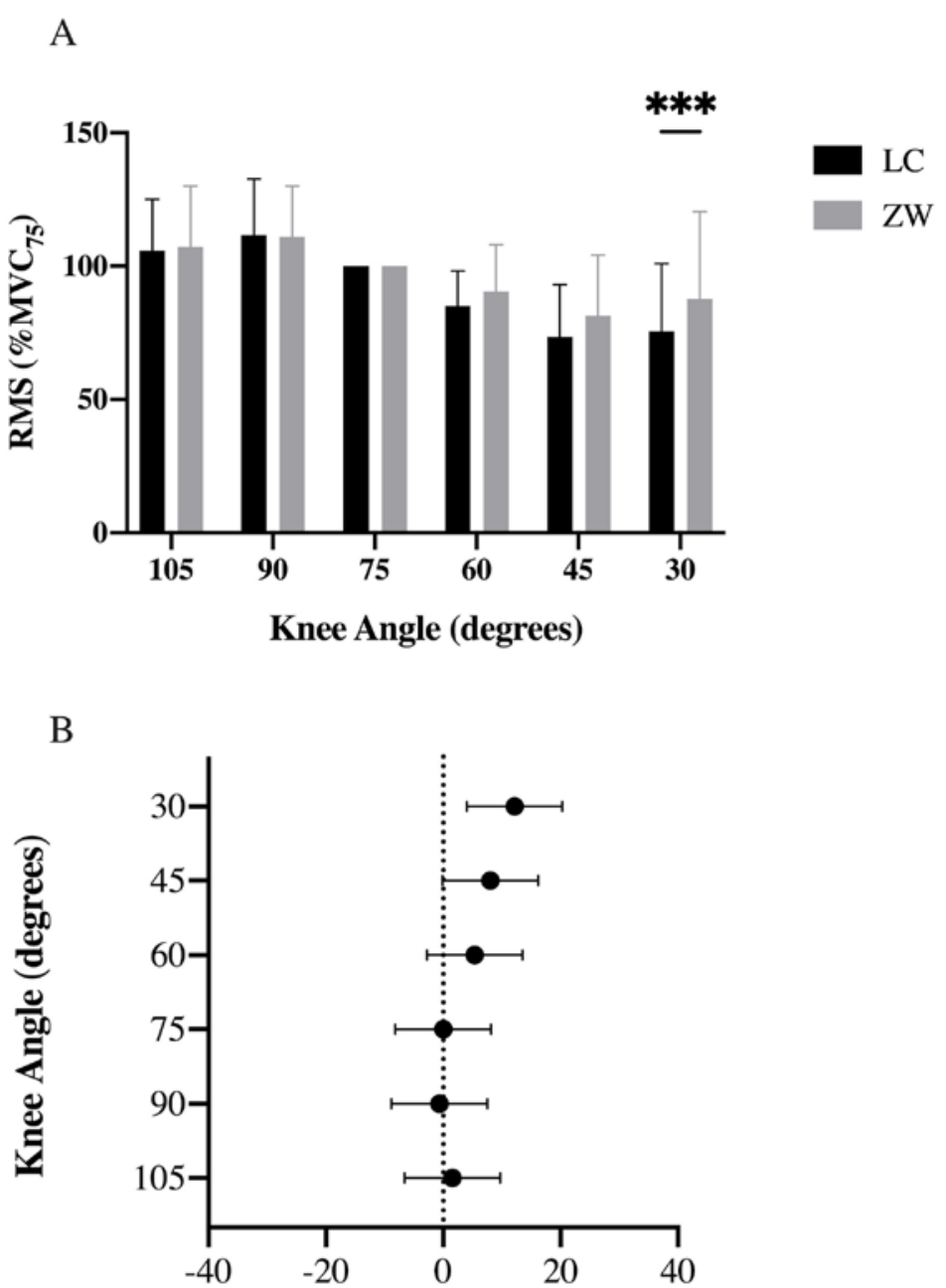

$\Delta$ RMS (ZW-LC)

Fig 1. Average sEMG activity (RMS at steady state) during maximal voluntary contractions at different knee angles as determined with a low-cost (LC) and a commercial (ZW) system (Panel A); data are normalized for the maximum sEMG activity recorded at $75 \%$ MVC. From 60 to $30^{\circ}$ of knee angle LC underestimatse the SEMG activity compared to $Z W$, but the difference is significant only at $30^{\circ}(P<0.05)$. The difference in RMS between the low-cost and commercial system is the largest at the most extended knee angle $\left(30^{\circ}\right.$; Panel $\left.B\right)$.

sEMG amplitude. These differences imply that ZW should be more sensitive and accurate as a function of the signal variation.

\section{Data analysys}

Data acquired with both systems were analyzed by a custom-made Matlab script (R2020a); data analysis was identical for the signals derived from both apparatuses. First, the DC offset was removed from the RAW signals (all contractions and for all devices): on the average, it was $347 \pm 2 \mathrm{mV}$ and $5 \pm 2 \mathrm{mV}$ for $\mathrm{ZW}$ and LC, respectively. The signals were then rectified and filtered with a bandpass filter (Butterworth $10-450 \mathrm{~Hz}$; $2^{\text {nd }}$ order). Finally, the RMS was calculated over the period of contraction with a moving window of 25 ms (see Figure
S2 of the supplementary material). ${ }^{14}$ For the maximal voluntary contractions (duration: $5 \mathrm{~s}$ ), both the peak and the mean value of the root mean square (RMS) during the last $2 \mathrm{~s}$ of the steady-state contraction was calculated and used as an indication of the maximal possible EMG activity during each MVC (see Figure 2). ${ }^{15}$ MVC was the highest at $75^{\circ}$ knee angle; thus, the RMS during the MVC at $75^{\circ}$ was used to normalize EMG activity at the other knee angles (as suggested by Lanza et al.). ${ }^{8,9}$ Therefore, the RMS was finally expressed as a percentage of the RMS collected during the MVC at $75^{\circ}\left(\% \mathrm{MVC}_{75}\right)$, for both EMG systems. For the explosive contractions (performed at $75^{\circ}$ angle only), the Hodges \& Bui algorithm was first used to find the onset and the end of each contraction, ${ }^{16}$ in order to calculate the activation 

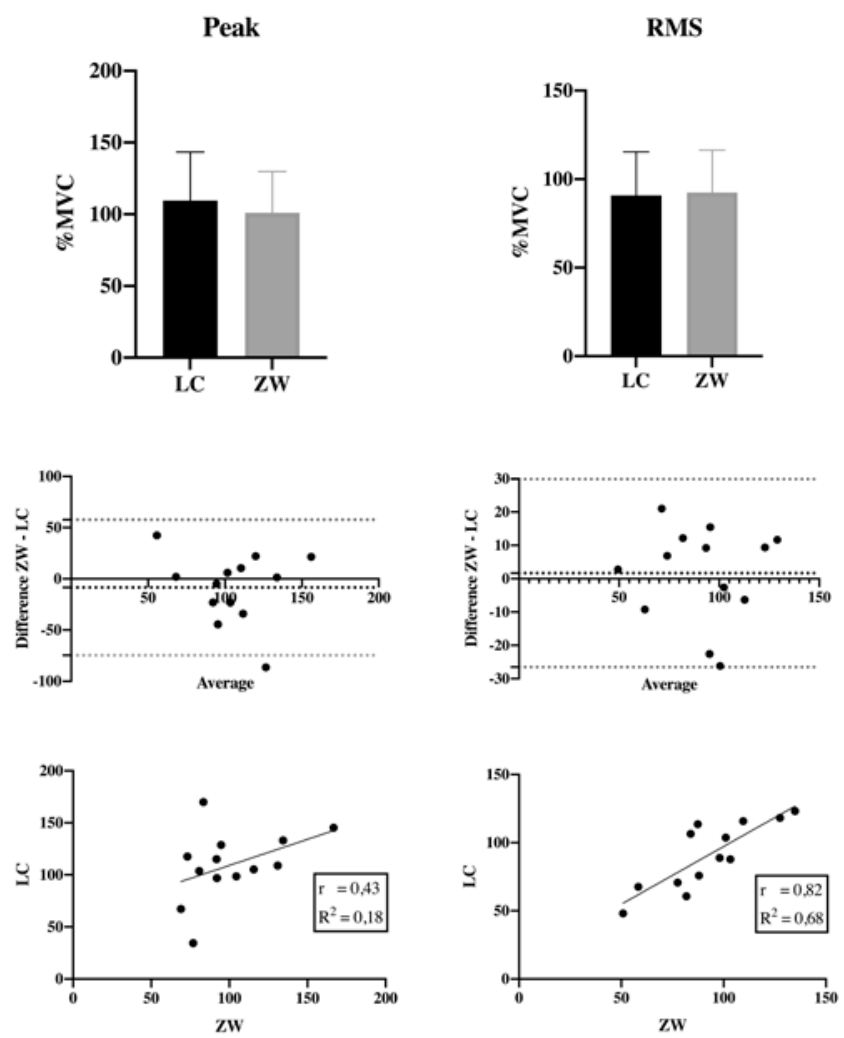

Fig 2. Mean and peak sEMG values (RMS) as determined with a low-cost (LC) and a commercial (ZW) system during fix-end explosive contractions are not significantly different (upper panel; values are mean $\pm S D$ ). However, bias and limits of confidence are much higher for peak than average values (Bland Altman plots, middle panel); moreover, the goodness of fit $\left(R^{2}\right)$ and the Pearson's correlation coefficient ( $r$ ) are, far larger for mean than peak values (only in the former case the correlation is significant) (bottom panel).

time (AT). The time to peak (TTP) was then calculated from the beginning of contraction to the first peak of the EMG signal ${ }^{1,3,17}$ (see Figure S3 of the supplementary material).${ }^{14}$ These procedures allow to compare the EMG devices in terms of temporal parameters. TTP and AT were not calculated for the MVCs because, in that case, the subjects were instructed to increase their force gradually. In terms of amplitude, the RMS peak and mean values were calculated during the activation period (see Figure 3) and then normalized to the maximum RMS value during the $\mathrm{MVC}$ at $75^{\circ}\left(\% \mathrm{MVC}_{75}\right)$, for both EMG systems.

\section{Statistics}

Data are presented as mean \pm SD. GraphPad Prism ${ }^{\circledR} 8$ (GraphPad Software, United States) was utilized for statistical analysis and figures preparation. For the concurrent validity, a range of correlational statistics explored the association between the two systems. Data referring to the explosive contractions (temporal and amplitude parameters) were evaluated by means of a paired t-tests ( $\mathrm{LC}$ vs. ZW). We considered the level P $<0.05$ as the cutoff value of significance. Data referring to the MVC at different knee angles were evaluated by means of a two-way repeated measured ANOVA (LC vs. ZW; $105^{\circ}, 90^{\circ}, 60^{\circ}, 45^{\circ}, 30^{\circ}$ ). Post-hoc tests (Bonferroni pairwise comparisons) were run to investigate for eventual differences (LC. vs ZW) at each angle. Also in this case the level $\mathrm{P}<0.05$ as the cutoff value of significance. Absolute agreement was explored using Bland Altman plots. ${ }^{18}$ Normality in data distribution was assessed by means of the Shapiro-Wilk test; since normality was observed for all the investigated parameters, the relative agreement was computed using Intra-class Correlation Coefficient (ICC). ${ }^{19}$ The determination coefficient $\left(\mathrm{R}^{2}\right)$ was utilized to evaluate the goodness of fit of relationship of a given parameter as assessed by means of both apparatuses.

\section{Results and Discussion}

The RMS values collected during MVCs and explosive fixed-end contraction, as well as the values of TTP and AT during the explosive fixed-end contractions are reported in Table 1 . In Table 2 the ICC values and the goodness of fit $\left(\mathrm{R}^{2}\right)$ between devices are reported for all the investigated variables.

As shown in Table 1, (normalized) RMS during the MVCs was found to differ between devices (LC vs. ZW, 
TTP
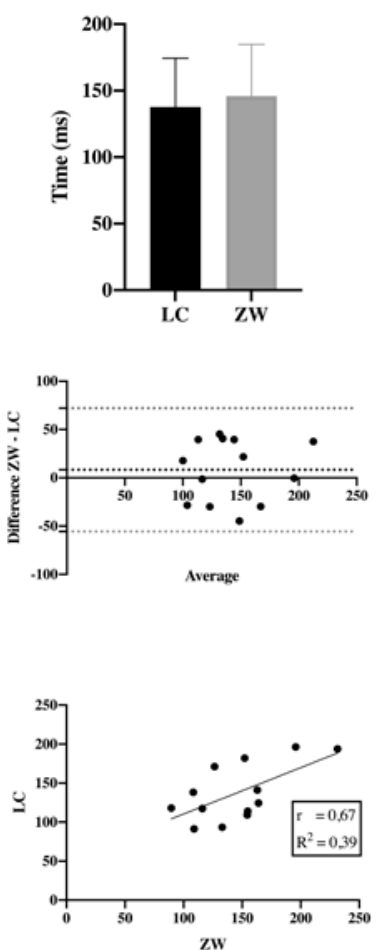

AT
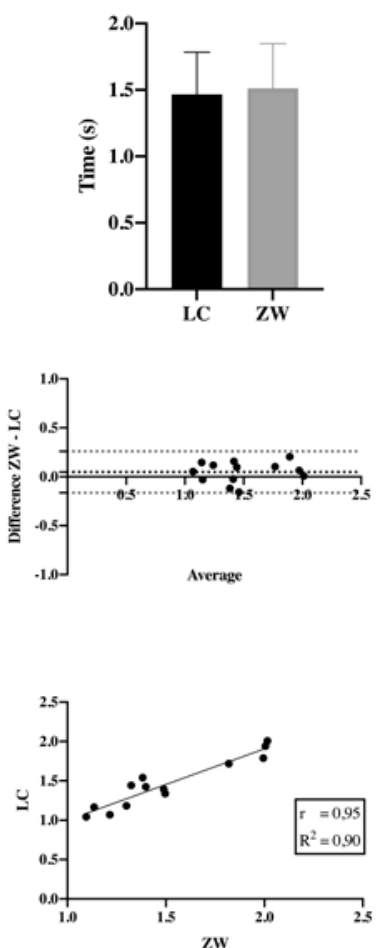

Fig 3. Time to peak (TTP) and activation time (AT) as determined with a low-cost (LC) and a commercial (ZW) system during fix-end explosive contractions are not significantly different (upper panel; values are mean $\pm S D)$. However, bias and limits of confidence are much higher for TTP than AT (Bland Altman plots, middle panel); moreover, the goodness of fit $\left(R^{2}\right)$ and the Pearson's correlation coefficient ( $r$ ) are, far larger for AT than TTP (only in the former case the correlation is significant) (bottom panel).

$\mathrm{P}<0.05)$ and among knee angles $(\mathrm{P}<0.05)$. A significant interaction (angle $\mathrm{x}$ device; $\mathrm{P}<0.05$ ) was also observed. Post hoc comparisons, however, indicate a significant difference only at $30^{\circ}$ of knee angle $(\mathrm{P}<0.001)$. As also indicated in Figure 1, the difference between the two systems is negligible when the knee is more flexed (75, 90 and $105^{\circ}$ ) and the average difference between LC and $\mathrm{ZW}$ data tends to increase from 60 to $30^{\circ}$. During the explosive fixed-end contractions at $75^{\circ}$ knee angle, no differences (paired t-test ) in peak and mean RMS, in TTP and in AT between the commercial and the low-cost system were observed (see Table 1). As shown in Table 2 , regarding the (normalized) RMS during the MVCs, the Bland-Altman analysis showed that the bias, SD and confidence intervals tend to increase from 60 to $30^{\circ}$. An excellent relative agreement (ICC) was observed between devices but at the most extended angle $\left(30^{\circ}\right)$ where ICC: $=0.71$. Goodness of fit followed the same trend: at $30^{\circ} \mathrm{R}^{2}$ is the lowest (0.55) whereas at the other knee angles $R^{2}$ ranged from 0.68 to 0.90 (see Table 2). Regarding the explosive fixed-end contraction, the Bland Altman analysis indicated a higher bias and larger confidence intervals for peak RMS compared to mean RMS (Figure 2 and Table 2). Accordingly, the goodness of fit is higher for mean RMS than for peak RMS $\left(\mathrm{R}^{2}=\right.$
0.68 and 0.18 , respectively) as is the case for the ICC. For peak RMS, ICC and $\mathrm{R}^{2}$ do not reach a significant level. Finally, the Bland Altman analysis indicated a higher bias and larger confidence intervals for TTP compared to AT (Figure 3 and Table 2). Accordingly, the goodness of fit is higher for activation time than for TTP $\left(\mathrm{R}^{2}=0.90\right.$ and 0.39 , respectively) as is the ICC.

In this study we assessed the concurrent validity of a custom-made (low-cost) and a commercial surface EMG apparatus in controlled experimental conditions, with the aim to investigate whether concurrent validity depends: i) on data acquisition and analysis; ii) on exercise type and intensity; iii) on the statistical analysis performed. We observed that, when data are derived from the same electrodes and analyzed with the same methods and procedures, the differences between the devices are fully explained by differences in their electronic components (gain and quality of the analog-digital converter). Furthermore, we observed no differences between systems in all the investigated variables when assessed by means of paired t-test and RM ANOVA, while BlandAltman analysis and correlation analysis (ICC and goodness of fit) highlight (similar) significant differences between devices. In turn, these differences were found to depend on exercise type (maximal voluntary contractions 
or explosive fix-end contractions) and intensity, with the low-cost device being less able in detecting rapid events (e.g. time to peak) compared to the commercial one. Several studies have shown that the relative alignment and movement of the muscle belly, the location of the innervation zone and many other structural factors could affect the sEMG signal. ${ }^{2,20}$ Moreover, different electrode position, as well as the inter-electrode distance, could change the quality of the signal. In this study both devices derived the signal from the same electrodes and, therefore, the observed differences could not be attributed to their positioning, as it could be the case in previous studies. ${ }^{7,8}$ Since the raw signals were analyzed and processed in the same way, the observed differences are mainly to be attributed to differences in the electronic components. As an example, during the MVC, the lowcost EMG device tends to underestimate sEMG activity but only at certain (extended) knee angles. More than to the knee angle per se, these differences could be attributed to differences in sEMG activity among conditions. Indeed, during MVC, sEMG activity decreases when extending the knee-joint (see figure 4A), as previously reported by Lanza et al. ${ }^{8,9}$ The commercial system has a larger gain (60 vs. $40 \mathrm{~dB}$ ) and a higherquality analog-digital converter (10 vs 16 bits) and is therefore more apt to correctly amplify the signal when the EMG activity is (relatively) low. This suggests that attention must be paid when using low-cost systems to investigate the sEMG signal during sub-maximal contractions. In regard to the explosive fix-end contractions, correlation analysis indicates that the mean RMS values and the time of activation are correctly measured by the low-cost system, but the time to peak and the peak RMS value are not. Also, these findings can be explained by differences in the electronic components: the lower signal gain and the lower analog digital converter quality imply a lower resolution of the low-cost device compared to the commercial one and this impedes to detect small signal fluctuations. This suggests that low-cost devices are not suitable to detect rapid EMG transient changes. This study provides new information about the validity of a low-cost apparatus in determining sEMG signal characteristics during exercise. Our data indicate that a custom-made apparatus could be utilized to detect timing and amplitude parameters during maximal exercise (MVC, in this study) but that attention should be payed when the EMG amplitude is (relatively) reduced (e.g. sub-maximal contractions) or during rapid changes in the sEMG signal [e.g. short-maximal (explosive) contractions].

In conclusion, because in our experimental conditions, the source of difference between the low-cost and commercial system can, essentially, be attributed to differences in gain settings and in the sampling rate (bit resolution) of the analog-digital converter, higher quality low-cost devices (e.g. newer version of Arduino with bigger CPU, higher clock and improved electronic interface) should be able to reduce the gap with commercial systems. This may lead to an increased use of sEMG analysis by sport scientists and/or physical therapists.

\section{List of acronyms}

LC - Low-cost

ZW - ZeroWire (commercial device)

sEMG - Surface Electromyography

MVC - Maximal voluntary contraction

TTP - Time to Peak

RMS - Root Mean Square

AT - Activation time

\section{Authors contributions}

PT contributed to the research concept and study design, literature review, data collection, statistical analyses, data analysis, data interpretation, manuscript writing and review. AM contributed to the study design, data collection, data interpretation, manuscript writing and review. PZ contributed to literature review, data interpretation, manuscript writing and review.

\section{Acknowledgments}

None.

\section{Funding}

This research received no external funding

\section{Conflict of Interest}

The authors declare no conflicts of interest.

\section{Ethical Publication Statement}

We confirm that we have read the Journal's position on issues involved in ethical publication and affirm that this report is consistent with those guidelines.

\section{Corresponding Author}

Andrea Monte, Department of Neurosciences, Biomedicine and Movement Sciences, University of Verona, Verona, Italy.

ORCID iD: 0000-0001-6604-2658

Andrea Monte: andrea.monte@univr.it

E-mails and ORCID iD of co-authors

Paolo Tecchio: paolo.tecchio@univr.it ORCID iD: 0000-0003-0371-8475

Paola Zamparo: paola.zamparo@univr.it ORCID iD: 0000-0002-6919-6721

\section{References}

1. Maffiuletti NA, Aagaard P, Blazevich AJ, Folland J, Tillin N, Duchateau J. Rate of force development: physiological and methodological considerations. 2016 Jun;116(6):1091-116. doi: 10.1007/s00421016-3346-6. Epub 2016 Mar 3.

2. Farina D, Cescon C, Merletti R. Influence of anatomical, physical, and detection-system parameters on surface EMG. Biol Cybern. 2002 Jun;86(6):445-56. doi: 10.1007/s00422-002-03092. 
3. Aagaard P, Simonsen EB, Andersen JL, Magnusson $P$, Dyhre-Poulsen $P$. Increased rate of force development and neural drive of human skeletal muscle following resistance training. J Appl Physiol (1985). 2002 Oct;93(4):1318-26. doi: 10.1152/japplphysiol.00283.2002.

4. Mills KR. The basics of electromyography. J Neurol Neurosurg Psychiatry. 2005 Jun;76 Suppl 2(Suppl 2):ii32-5. doi: 10.1136/jnnp.2005.069211.

5. Fuentes Del Toro S, Wei Y, Olmeda E, Ren L, Guowu W, Díaz V. Validation of a Low-Cost Electromyography (EMG) System via a Commercial and Accurate EMG Device: Pilot Study. Sensors (Basel). 2019 Nov 28;19(23):5214. doi: 10.3390/s19235214.

6. Supuk TG, Skelin AK, Cic M. Design, development and testing of a low-cost sEMG system and its use in recording muscle activity in human gait. Sensors (Basel). 2014 May 7;14(5):8235-58. doi: 10.3390/s140508235. Erratum in: Sensors (Basel). 2014;14(8):15639-40.

7. Heywood S, Pua YH, McClelland J, Geigle P, Rahmann A, Bower K, Clark R. Low-cost electromyography - Validation against a commercial system using both manual and automated activation timing thresholds. J Electromyogr Kinesiol. 2018 Oct;42:74-80. doi: 10.1016/j.jelekin.2018.05.010. Epub 2018 Jun 2.

8. Lanza MB, Balshaw TG, Folland JP. Do changes in neuromuscular activation contribute to the knee extensor angle-torque relationship? Exp Physiol. 2017 Aug 1;102(8):962-973. doi: 10.1113/EP086343. Epub 2017 Jul 12.

9. Lanza MB, Balshaw TG, Folland JP. Explosive strength: effect of knee-joint angle on functional, neural, and intrinsic contractile properties. Eur J Appl Physiol. 2019 Aug;119(8):1735-1746. doi: 10.1007/s00421-019-04163-0. Epub 2019 May 21.

10. Bakenecker P, Zinke F, Hahn D. Explosive torque capacity and fascicle behavior during accelerated concentric contractions. Scand J Med Sci Sport 2016. https://doi.org/10.1111/sms.12812.

11. Hahn D, Bakenecker P, Zinke F. Neuromuscular performance of maximal voluntary explosive concentric contractions is influenced by angular acceleration. Scand J Med Sci Sports. 2017 Dec;27(12):1739-1749. doi: 10.1111/sms.12812. Epub 2016 Dec 28.
12. Monte A, Baltzopoulos V, Maganaris CN, Zamparo P. Gastrocnemius Medialis and Vastus Lateralis in vivo muscle-tendon behavior during running at increasing speeds. Scand J Med Sci Sports. 2020 Jul;30(7):1163-1176. doi: 10.1111/sms.13662. Epub 2020 Apr 13.

13. Arampatzis A, Karamanidis K, De Monte G, Stafilidis S, Morey-Klapsing G, Brüggemann GP. Differences between measured and resultant joint moments during voluntary and artificially elicited isometric knee extension contractions. Clin Biomech (Bristol, Avon). 2004 Mar;19(3):277-83. doi: 10.1016/j.clinbiomech.2003.11.011.

14. Supplementary material available at: https://drive.google.com/file/d/1rA4ZJz1CE9tPU0 32nURuwE0cr1iWwb6o/view?usp=sharing

15. Hermens HJ, Freriks B, Merletti R, Stegeman D, Blok J, Rau G, et al. European Recommendations for Surface ElectroMyoGraphy. Roessingh Res Dev. 1999;8-11.

16. Hodges PW, Bui BH. A comparison of computerbased methods for the determination of onset of muscle contraction using electromyography. Electroencephalogr Clin Neurophysiol. 1996 Dec;101(6):511-9. doi: 10.1016/s00134694(96)95190-5.

17. Folland JP, Buckthorpe MW, Hannah R. Human capacity for explosive force production: neural and contractile determinants. Scand J Med Sci Sports. 2014 Dec;24(6):894-906. doi: 10.1111/sms.12131. Epub 2013 Oct 29.

18. Bland JM, Altman DG. Statistical methods for assessing agreement between two methods of clinical measurement. Lancet. 1986 Feb 8;1(8476):307-10.

19. Fleiss JL. The Design and Analysis of Clinical Experiments. Biometrical Journal. New York: John Wiley \& Sons; ; 1986. http://doi.wiley.com/10.1002/bimj.4710300308.

20. Rainoldi A, Nazzaro M, Merletti R, Farina D, Caruso I, Gaudenti S. Geometrical factors in surface EMG of the vastus medialis and lateralis muscles. J Electromyogr Kinesiol. 2000 Oct;10(5):327-36. doi: 10.1016/s1050-6411(00)00024-9.

Submission: March 08, 2020

Revision received: April 02, 2020 Accepted for publication: April 02, 2021 